\title{
Resolução de uma Equação Diferencial Parcial pelo Método de Separação de Variáveis no problema de Condução de Calor em uma Barra
}

\author{
Gonzaga, B. R. ${ }^{*}$; Ramos, G. B.G²; Loeffler, C.F³. \\ 1 Instituto Federal do Espírito Santo, Vitória, ES, Brasil. \\ 2 Instituto Federal do Espírito Santo, Vitória, ES, Brasil. \\ 3 Programa de Pós-Graduação em Engenharia Mecânica, Universidade Federal do Espírito Santo, Vitória, ES, \\ *e-mail: bgonzaga.mat@gmail.com
}

\section{Resumo}

Este artigo é fruto de dois trabalhos apresentados oralmente no ano de 2013, sendo o mesmo aperfeiçoado em cada ano com novos dados, em especial na presente proposta se encontra a solução tridimensional. Então, o objetivo deste trabalho é gerar uma solução analítica de um problema não homogêneo de Condução de Calor numa Barra, que possa servir de referência para outras aplicações científicas. Em geral, Equações Diferenciais Parciais com condições de contorno não homogêneas, se resolve, reduzindo a mesma a um problema com condições de contorno homogêneas. Sabe-se que uma posterior avaliação com recursos computacionais, ou até mesmo estimativa de convergência, são extremamente uteis em alguns modelos. Então, o método da separação de variáveis quando aplicado na condução de calor, equação da onda ou equação do potencial, e posteriormente analisados de forma numérica ou gráfica, obtém-se um solução com melhor qualidade na interpretação de dados nos diversos segmentos da ciência.

Palavras-Chave: Método de Separação de Variáveis, Problemas Não Homogêneos, Resposta da temperatura.

\section{Abstract}

The objective of this work is to generate an analytical solution of an inhomogeneous problem of heat conduction in a bar that can serve as reference for other scientific applications. In general, Partial Differential Equations with inhomogeneous boundary conditions, is solved by reducing it to a problem with homogeneous boundary conditions. Further evaluation with computer resources, or even estimate convergence, are extremely useful models. Then, the method of separation of variables when applied to the heat conduction, the potential wave equation or equation, and then analyzed in numerical or graphical form, gives a solution with better quality in data interpretation in the various segments of science.

Keywords: Variable Separation Method, No Problems Homogeneous, temperature response. 


\section{Introdução}

O problema consiste na condução de calor em uma barra, feita com material homogêneo [1]. Suponha que a barra tenha $30 \mathrm{~cm}$ de comprimento para a qual $\alpha^{2}=1_{x}$ onde $\alpha^{2}$ é a constante de difusividade térmica. A distribuição de temperatura é dada pela condição inicial $u\left(x_{v}, 0\right)=60-2 x$ e que as condições de contorno são $u\left(0_{x} t\right)=20$ e $u(30, t)=50$. É provável que depois de algum tempo, quando, será alcançada uma temperatura estacionária $v(x)$, que é independente do tempo t e das condições iniciais. Então, vamos tentar expressar $u\left(x_{v} t\right)$ como a soma da distribuição de temperatura estada estacionário com outra distribuição transiente $w\left(x_{z} t\right)$. A demonstração da equação diferencial (1) foge ao escopo deste trabalho, mas, podemos afirmar que tal demonstração é feita a partir de uma situação ou processo físico do fenômeno em questão.

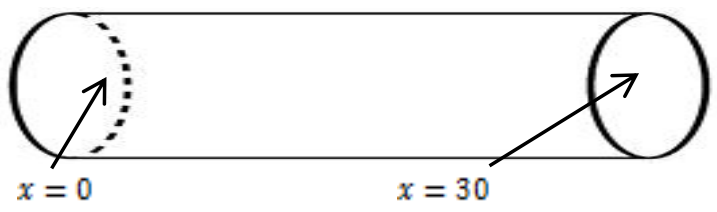

Figura 1: Uma Barra sólida condutora de calor

Da equação de calor temos:

$$
\alpha^{2} u_{x x}=u_{t}
$$

\section{Aplicação do Método de Separação}

\section{de Variáveis}

De acordo com a estratégia do Método de Separação de Variáveis para problemas nos quais as condições de contorno não são homogêneas, é necessário supor uma solução do tipo:

$$
u\left(x_{v} t\right)=v(x)+w\left(x_{v} t\right)
$$

Como $v(x)$ tem que satisfazer a equação do calor (1), temos que $v^{t s}(x)=0$. Assim, com as devidas condições de contorno $v(0)=20$ e $v(30)=50$, logo $v(x)=20+x$.

A solução transiente é dada por

$$
w_{x x}=w_{t}
$$

Logo, temos as seguintes condições de contorno homogêneas e condição inicial modificada:

$$
\begin{aligned}
& W(0, t)=0 \\
& W(30, t)=0
\end{aligned}
$$

$w\left(x_{v} 0\right)=60-2 x-(20+x)=40-3 x$

Observa-se que agora temos um problema de condições de contorno homogêneas para ser resolvido, com $\alpha^{2}=1$ e $f(x)=40-3 x$. Então, será preciso resolver o problema pelo método de separação de variáveis, sendo:

$$
w\left(x_{v} t\right)=X(x) T(t)
$$

Substituindo $w$ dado pela equação (4) na equação (1), obtemos

$$
\alpha^{2} X^{t r} T=X T
$$

A equação (5) é equivalente a:

$$
\frac{X^{n}}{X}=\frac{1}{\alpha^{2}} \frac{T^{x}}{T}
$$

Então, a equação (6) deve ser igual a uma constante, pois, é o único modo de estabelecer uma igualdade entre derivadas espaciais e temporais [2]. Assim, obtemos:

$$
\frac{x^{n}}{X}=\frac{1}{\alpha^{2}} \frac{T^{x}}{T}=-\gamma, \text { onde } \alpha^{2}=1
$$

Obtemos, então, as duas equações ordinárias a seguir para $X(x)$ e $T(t)$ :

$$
\begin{aligned}
& X^{s s}+\gamma X=0 \\
& T^{s}+\gamma T=0,
\end{aligned}
$$

Agora, vamos considerar a solução da Eq. (8) que satisfazem as condições de contorno da função espacial-temporal, onde $w(0, t)=w(30, t)=0$. As únicas soluções não triviais são as autofunções:

$$
X_{n}(x)=\operatorname{sen}\left(\frac{n \pi x}{a 0}\right), n=1,2,3_{x a}
$$

Associadas aos autovalores:

$\gamma_{n}=\frac{n^{2} \pi^{2}}{900}, n=1,2,3, \ldots$

Voltando para a Eq. (9)para $T(t)$ e substituindo $\gamma_{n}$ por $\frac{n^{2} \pi^{2}}{900}$, temos:

$\mathrm{T}^{\prime}+\left(\frac{\mathrm{n}^{2} \mathrm{\pi}^{2}}{900}\right) T=0$,

$\operatorname{logo} T(t)$ é proporcional a:

$$
\exp \left(\frac{-n^{2} \pi^{2} t}{900}\right) \text {. }
$$


Portanto, multiplicando as soluções das equações (8) e (9), obtemos:

$$
\begin{aligned}
& W\left(x_{v} t\right)=c_{\mathrm{n}} e^{\frac{-\mathrm{H}^{2} \mathrm{n}^{2} \mathrm{r}}{200}} \operatorname{sen}\left(\frac{\mathrm{n \pi x}}{\mathrm{a} 0}\right), \mathrm{n}=1,2,3, \ldots \\
& \text { Onde } c_{\mathrm{n}}=\frac{2}{\mathrm{a} 0} \int_{0}^{\mathrm{a0}}(40-3 x) \operatorname{sen}\left(\frac{n \pi x}{\mathrm{a} 0}\right) d x
\end{aligned}
$$

Assim, $u\left(x_{v} t\right)=20+x+\sum_{n=1}^{m} c_{n} e^{\frac{-m^{2} n^{2} r}{200}} \operatorname{sen} \frac{n n x}{a 0}$, onde $c_{\mathrm{n}}$ são os termos da Eq. (13).

Calculando os dois primeiros termos da função da Equação (13) [3], temos:

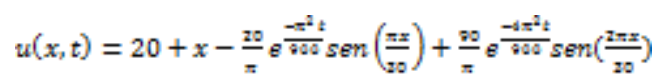

\section{Resultados}

Encontrada a solução analítica, pode-se construir gráficos da equação (16), a fim de validar o método, bem como discutir os resultados. Então, faz-se primeiro o estudo para a condição inicial .

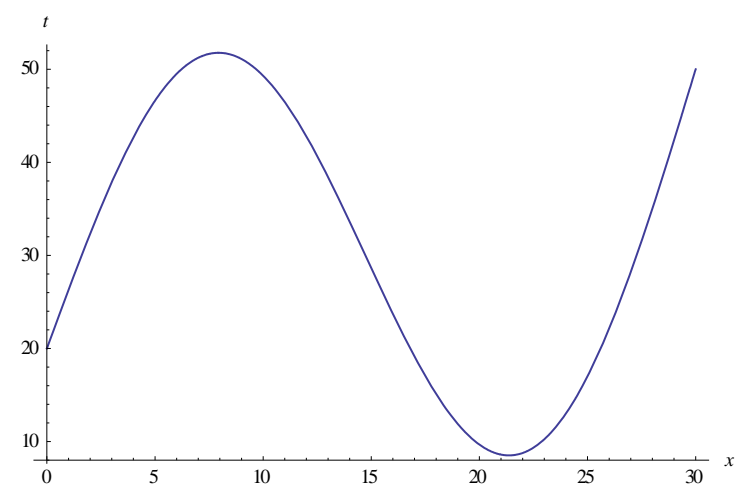

Figura 2: Gráfico da equação (16) para t $=0$

Verifica-se que a solução obedece à condição inicial $u\left(x_{0} 0\right)=20$ e $u\left(x_{v}, 30\right)=50$. Então, estuda-se a solução para valores de $t \geq 0$. Logo, aplica-se $t=30$, na equação (16) e obtem-se a figura 3 :

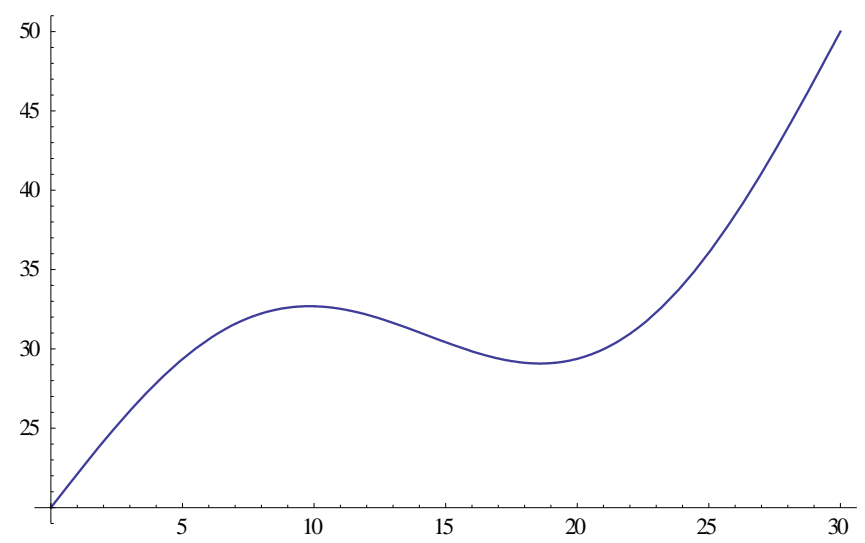

Figura 3: Gráfico da equação (16) para $t=30 \mathrm{~s}$.

Observa-se, que na posição $x=10$, a barra apresenta uma temperatura pouco maior que 30 graus, enquanto que ocorre uma preservação das condições de contornos $u\left(0_{0}, t\right)=20$ e $u\left(30_{0} t\right)=50$. Espera-se que a partir de dado instante, o problema fique no modo estacionário, ou seja, a temperatura será regida por uma função linear. Então, estuda-se a função e esboça-se o gráfico para .

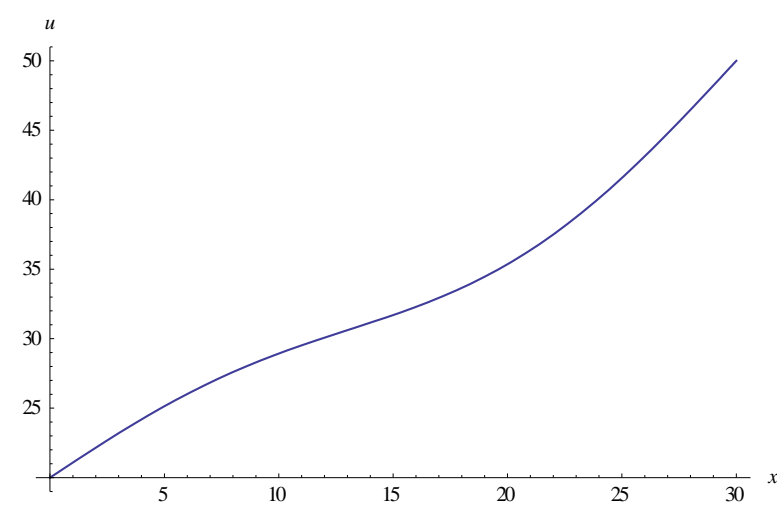

Figura 4: Gráfico da equação (16) para $t=60$,

Observa-se que na posição $x=10$, ocorreu uma queda na temperatura, sendo aproximadamente 28 graus. Verifica-se também que na posição $x=20$, ocorre um aumento de temperatura em relação ao gráfico anterior, sendo agora, próxima dos 35 graus. Então, a fim de verificar o modo estacionário do problema, aplica-se o valor de $t=300$, e tem-se: 


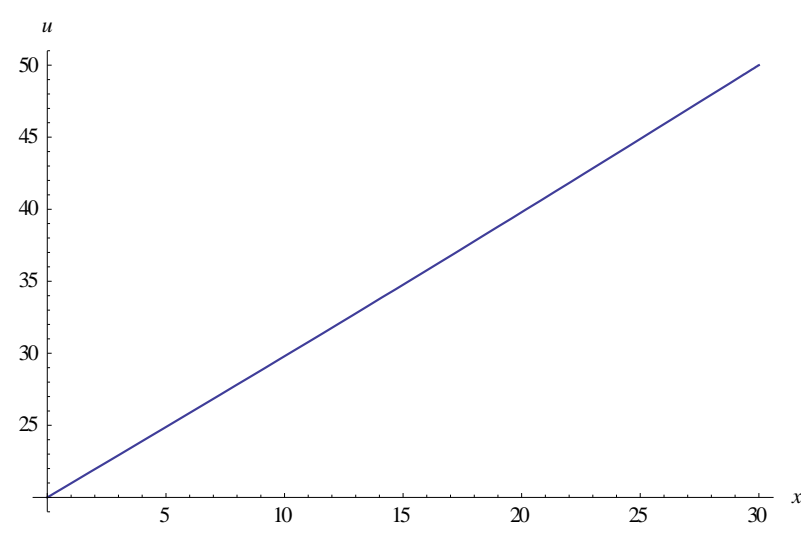

Figura 5: Gráfico da equação (16) para $\mathrm{t}=300$.

Observa-se que a temperatura estaciona em cada posição, assim, a equação da reta que se ajusta ao modelo é dada por $\mathfrak{v}(x)=20=x$. Podemos comparar o gráfico da figura 5 , com uma solução tridimensional, assim, a figura 6 , mostra claramente os valores das temperaturas representadas no eixxo $u$ em consonância com os que foram encontrados na figura 4. Além disso, a visualização tridimensional permite verificar 0 comportamento da solução de forma mais rápida e objetiva, inferir pontos de máximo e mínimo, descontinuidades e pontos onde a função pode ou não ser derivável e etc. Então, na solução analítica representada pela equação (16), e fazendo $0 \leq t \leq 300$ e $0 \leq x \leq 30$, tem-se:

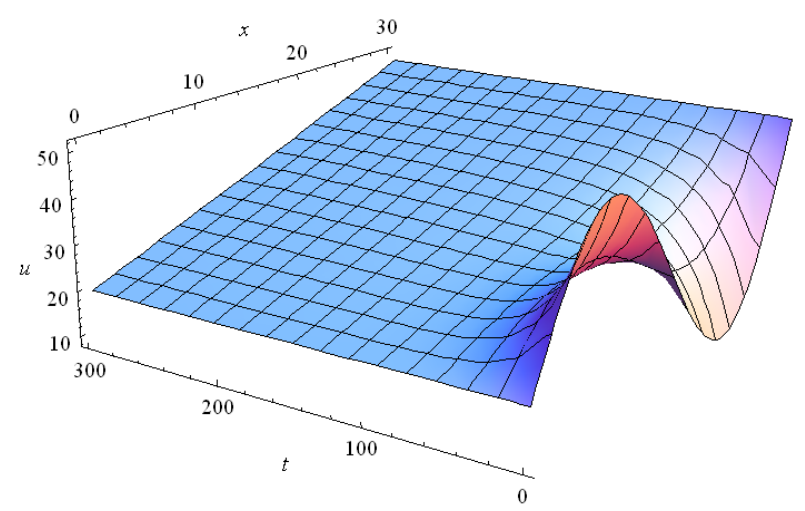

Figura 6: Gráfico tridimensional da equação (16), com $0 \leq t \leq 300$.

\section{Conclusões}

Resalta-se a eficiência do método de separação de variáveis, para problemas com condições de contorno não homogêneas, contudo, os métodos numéricos podem e devem ser usados para equações diferenciais de alto grau de complexidade, de modo que a hegemonia dos métodos discretos de solução é completamente indiscutível e teste de validação realizado pela comparação de resultados entre métodos distintos é a tática mais adequada em um contingente cada vez maior de casos [4]. Todavia, soluções analíticas enriquecem o debate, e forçam os pesquisadores a cada vez mais aperfeiçoarem os métodos discretos. Em nosso presente trabalho, os gráficos foram gerados com uso do software Wolfram Mathematica 9.0, possibilitando assim, uma maior eficiência de nossos dados. Didaticamente, o problema da condução de calor é útil para explicar a variação de temperatura de acordo com o tempo e o espaço, além disso, permite na prática, visualizar o modo estacionário a partir de certo instante.

\section{Referencia}

\section{Livro}

[1] Boyce. W, DiPrima.R.C, "Equações Diferenciais Elementares e Problemas de Valor de Contorno", LTC Editora, Rio de Janeiro, 2010.

\section{Trabalho Oral Apresentado}

[2] GONZAGA, B. R., LOEFFLER, C.F. Resolução de uma Equação Diferencial Parcial pelo Método de Separação de Variáveis no problema de Condução de Calor numa barra. Aprovado e Apresentado no IV Encontro de Física Aplicada 02 a 06 de Junho de 2013

[3] GONZAGA, B. R., LOEFFLER, C.F. Resolução de uma Equação Diferencial Parcial pelo Método de Separação de Variáveis no problema de Condução de Calor numa barra. Aprovado e Apresentado no Congresso de matemática Aplicada e Computacional 23 a 27 de Setembro de 2013.

\section{Teses ou dissertações}

[4] Solução Analítica de um Problema Bidimensional de propagação de ondas em meio não homogêneo pelo Método de separação de variáveis. Dissertação (Mestrado em Ciências Mecânicas), Programa de PósGraduação em Engenharia Mecânica, Universidade Federal do Espírito Santo, Vitória, 2014. 